\title{
Memory impairment in rats with glutamatergic temporal systems disrupted is attenuated by D-cycloserine: Effects of postoperative time of injection
}

\author{
TROND MYHRER and RAGNHILD E. PAULSEN \\ Norwegian Defence Research Establishment, Division for Environmental Toxicology \\ Kjeller, Nomway
}

\begin{abstract}
Disconnection of the neural fibers between the temporal cortex (TC) and the lateral entorhinal cortex (LEC) in rats causes impaired memory and reduced concentration of glutamate in both the TC and the LEC. Administration of the glutamatergic agonists NMDA, glycine, or AMPA improves retroactive memory, which is more severely impaired by TC/LEC lesions than proactive memory in a three-choice brightness discrimination task. It has not been determined, however, whether there are positive effects of agonists given at a particular time of injection. The purpose of the present study was to address this issue by applying (on Postoperative Days 0-12) the partial agonist D-cycloserine, which may be of special clinical interest. The results show that injections given shortly after surgery (Days 0-1) or just prior to retrieval (Day 12) are much more effective than injections given between these extremes. Injections given on Postoperative Day 8 had no effect at all, whereas a stepwise increase in effects was seen toward both Day 0 and Day 12. It is suggested that the action profile of cycloserine may reflect effects of both functional and pharmacological mechanisms. Cycloserine may represent a relevant means of treatment of dementia.
\end{abstract}

The temporal region of the rat is a critical area for mnemonic processing. Lesions of the temporal cortex (TC), the lateral entorhinal cortex (LEC), or the fiber connections between these areas impair memory functions. In particular, it has been shown that TC/LEC disruptions cause marked impairment of both retroactive memory (retrograde amnesia) and proactive memory (anterograde amnesia; Myhrer, 1991, 1992). At least some of the disrupted neurons use glutamate as a neurotransmitter, because reduced high-affinity $\mathrm{D}$-aspartate uptake in both the TC and the LEC follows TC/LEC transsections (Myhrer, Iversen, \& Fonnum, 1989). An assumption of glutamatergic involvement in mnemonic processes in the temporal region receives support from the finding that administration of glutamatergic agonists can completely restore memory function in rats with TC/LEC lesions. Retroactive memory, which is most severely impeded by TC/LEC lesions, is completely ameliorated by i.p. injections of NMDA, glycine, or AMPA, whereas administration of kainic acid only produces a slight improvement of memory (Myhrer \& Paulsen, 1992).

In a subsequent study, effects of dose and time of glycine injections on proactive memory were examined.

This research was supported by the Research Council of Norway, Grant 326.93/010 to T.M. Knut K. Skrede is gratefully acknowledged for fruitful discussions during the preparation of this manuscript. Correspondence should be addressed to T. Myhrer, Norwegian Defense Research Establishment, Division for Environmental Toxicology, P.O. Box 25, N-2007 Kjeller, Norway.
In the proactive paradigm, rats with TC/LEC lesions displayed a slight mnemonic deficit during acquisition of a discrimination task (Myhrer, 1992). When one large dose $(750 \mathrm{mg} / \mathrm{kg}$ ) of glycine was given immediately prior to acquisition, both acquisition and retention occurred at normal rate in TC/LEC animals. A large dose of glycine administered immediately after acquisition or just prior to retrieval fully restored retention, whereas a large dose given midway between learning and retention did not improve retention significantly (Myhrer, Johannesen, \& Spikkerud, 1993).

In the study of retroactive memory (Myhrer \& Paulsen, 1992), one injection of agonist was given immediately after completion of the surgery and an additional injection followed 2 days after surgery in an attempt to enhance neural activity necessary for retaining relevant information. Some animals also received a third injection 10 days after surgery, but this injection did not seem to have any effect. From this study of retroactive memory, it was not possible to determine whether the positive effects of agonists were dependent on a particular time of injection.

Because potent agonists like glycine may run the risk of causing further damage by excitotoxic effects, partial agonists may represent an appropriate alternative in a therapeutic sense. For this reason, the partial agonist D-cycloserine has been suggested as a relevant relief measure to compensate for the declining cognitive capability in patients with Alzheimer's disease (Francis, Pangalos, \& Bowen, 1992). Cycloserine freely crosses the 
blood-brain barrier (Monahan, Handelmann, Hood, \& Cordi, 1989) and has about $60 \%$ of the maximal response of glycine (Huettner, 1991). This partial agonist has been shown to be a positive modulator of the glycine site at the NMDA receptor (Monahan et al., 1989).

The purpose of the present study was twofold. First, it was intended to ascertain whether cycloserine might mitigate the pronounced retrograde impairment seen in rats with TC/LEC lesions. Second, it was intended to determine whether there is a critical time for administration of the agonist in order to obtain optimal effect. To highlight these issues, 13 groups of rats with TC/LEC lesions were used, and each group received a single injection of cycloserine on one of the days between surgery and testing for retention (Postoperative Days 0-12). These groups, in addition to 3 groups of saline-treated TC/LEC rats and 3 groups of saline-treated control rats, were tested in a three-choice brightness discrimination task used in previous studies.

\section{METHOD}

\section{Subjects}

Seventy-six male Wistar rats from a commercial supplier (Møllegaard Breeding Laboratories), weighing 280-310 g at the time of surgery, served as subjects. They were randomly assigned to three categories of treatment: lesion and saline, lesion and cycloserine, or saline only. Three groups of rats (each consisting of 4 rats) received bilateral TC/LEC lesions and a single injection of saline on Postoperative Day 0,6 , or 12 , respectively. Thirteen groups of rats (each consisting of 4 rats) received bilateral TC/LEC lesions and a single injection of cycloserine on one of the postoperative days between 0 and 12 . Three groups of rats (each consisting of 4 rats) underwent sham surgery and received a single injection of saline on Postoperative Day 0, 6, or 12, respectively. The rats were housed individually and had free access to commercial rat pellets and water. The animals were handled individually 4 days preoperatively, being allowed to explore a table top $(80 \times 60 \mathrm{~cm})$ for $3 \mathrm{~min}$ a day. The climatized $\left(21^{\circ} \mathrm{C}\right)$ vivarium was illuminated from 0700 to $1900 \mathrm{~h}$.

\section{Surgery}

The rats were anesthetized i.p. with diazepam $(10 \mathrm{mg} / \mathrm{kg})$ and fentanyl fluanisone $(2 \mathrm{mg} / \mathrm{kg})$ and placed with their skulls horizontal in a stereotaxic head holder. The bilateral lesions were made mechanically by means of the sharp edges of cannulas (diameter, $0.5 \mathrm{~mm}$ ) provided with a collar to control for insertion depth. The cannula to be used was mounted on a syringe. The point of insertion was $7.8 \mathrm{~mm}$ posterior to bregma and $6.7 \mathrm{~mm}$ lateral to midline. Each cannula was inserted into the brain in a position deviatiing $20^{\circ}$ from the vertical in the sagittal plane (tip of cannula pointing rostrally). From this position, the syringe was moved seven times back and forth in an axis deviating about $45^{\circ}$ from the frontal plane (opening of angle pointing medially). These maneuvers were carried out in two stages, with insertion depths 6 and $8 \mathrm{~mm}$ from the top of the skull. In this way, the distal part of the angular bundle was transected at a site corresponding approximately to the level of the rhinal fissure.

\section{Histology}

Upon termination of testing, the animals were decapitated, and the brains were removed and frozen. The brains were sectioned horizontally on a $\mathrm{CO}_{2}$ freezing microtome at $30 \mu \mathrm{m}$, with every twelfth section being preserved. The sections were stained with methylene blue. The extent of fibers transected was estimated from the degree to which the white matter between the TC and LEC was damaged at the three dorsoventral levels presented in Figure 1. The white matter (not the alveus) was divided in four equal columns, each column representing $25 \%$ of the fibers. The occurrence of damage was evaluated under relatively high magnification. The number of columns affected at each dorsoventral level was counted, and the mean percentage of damage was computed for each animal.

\section{Drugs}

D-Cycloserine (purchased from Sigma Chemical Co., St. Louis) was dissolved in $0.9 \%$ physiological saline; $\mathrm{pH} 6$. The dose injected i.p. was $3 \mathrm{mg} / \mathrm{kg}$ (Monahan et al., 1989). Because neutral or acid solutions of cycloserine have been reported to be unstable (Merck Index, 1989), the solutions were stored for not more than 1 week at refrigerator temperature. Physiological saline was injected i.p. in a dose of $0.5 \mathrm{ml}$.

\section{Apparatus}

Testing of simultaneous brightness discrimination was carried out in a Plexiglas cage $(56 \times 34 \times 20 \mathrm{~cm})$ described previously (Myhrer \& Nævdal, 1989). In brief, a Plexiglas wall with an opening $(10 \times 10 \mathrm{~cm})$ in the middle divided the apparatus in two equal compartments: the start compartment and the goal compartment. Three interchangeable aluminum cylinders $(3 \times 7 \mathrm{~cm})$ with a round well $(2 \times 2 \mathrm{~cm})$ in the top served as discriminanda. The cylinders were located in fixed positions (equidistant from each other) along the wall opposite to the partition wall in the goal compartment. The cylinders were natural gray (aluminum) or painted black (except for the well). The well of the positive cylinder was filled with water. The only light was a $15-\mathrm{W}$ bulb $60 \mathrm{~cm}$ above the apparatus.

\section{Procedure}

During acquisition and retention testing, the rats were deprived of water for $23.5 \mathrm{~h}$ a day. Prior to acquisition, each rat was allowed to explore the empty test apparatus for $5 \mathrm{~min}$ and then underwent pretraining. The pretraining consisted of running from the start compartment into the goal compartment, in which the rats were rewarded with some laps of water from a glass dish. Each rat received 5 trials. On the 1 st day of acquisition, the subjects were trained to discriminate between the cylinders and received some laps of water from the well in the positive cylinder. The rats were given 10 trials; the intertrial interval, during which they stayed in the home cage, was $20 \mathrm{sec}$. On the 2 nd day, the animals were given trials until the occurrence of five correct responses in succession. Because the task is easy to learn, the learning criterion was set low to avoid overlearning.

The rats were operated on $24 \mathrm{~h}$ after the learning criterion had been achieved. Twelve days following surgery, the animals were tested for retention of the discrimination task. Testing was terminated when the previous criterion was reached. The following behaviors were recorded: number of trials to criterion, number and type of errors to criterion. In order to drink or investigate whether the well in a cylinder contained water, the rats had to stand on their hind legs with at least one forepaw on top of the cylinder. Error response was scored when a negative cylinder was mounted and found empty of water (e.g., licking the empty well). Approaching or investigating negative cylinders (except the well) was not scored as an error. The positive cylinder was either black or gray, and the two cylinders of opposite color were negative. The position of the positive cylinder (left, middle, right) was changed according to a prearranged randomized order. One set of randomized positions was used on the 1 st day of training and another one on the 2nd day and on retention testing. A counterbalanced paradigm was followed in which half of the subjects were trained with the black 

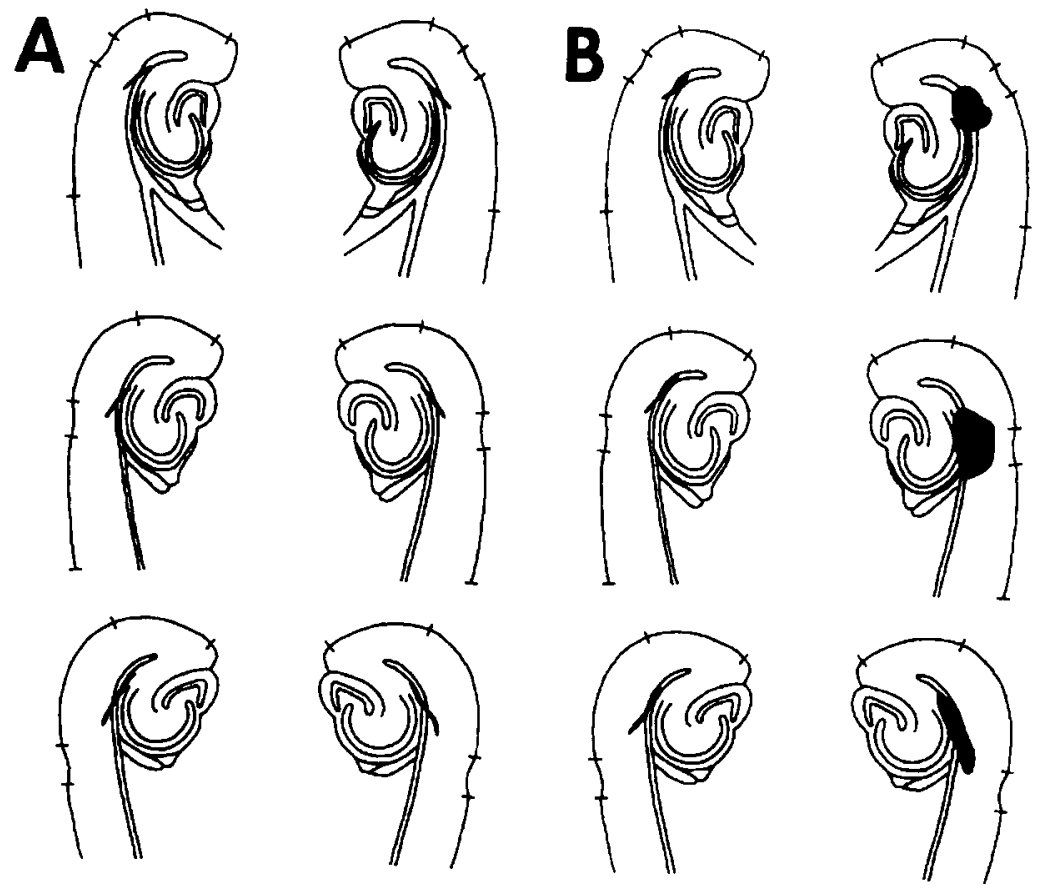

Figure 1. Reconstruction of brain sections indicating locations of lesions in three levels of horizontal sections (distance between each section, $1.5 \mathrm{~mm}$ ). (A) Typical lesion in salinetreated animals; (B) typical lesion in cycloserine-treated animals.

cylinder as positive and the other half with the gray cylinder as positive.

During the initial phase of learning this task, the rats frequently put their snouts close to negative cylinders and then left them. Because olfactory cues are of no guidance in this respect, the rats most likely responded to the color. An approach to a positive cylinder was immediately followed by rearing and drinking from the well. As training proceeded, rats gradually ceased approaching negative cylinders and headed for the positive cylinder when entering the goal compartment. It is not likely that they changed their learning strategies at this stage of training by addressing the positive cylinder because of its odd appearance (one positive vs. two negative cylinders), since approaching negative cylinders did occur now and then.

\section{Statistics}

Statistical overall analyses were made with one-way analyses of variance (ANOVAs) and group comparisons with Tukey-Kramer post hoc tests or two-tailed $t$ tests. Computations were carried out with the InStat system, a statistical software program (GraphPad Software, San Diego, CA).

\section{RESULTS}

\section{Histology}

The TC/LEC lesions appeared as a section through the white matter at a site between TC and LEC (Figures 1 and 2). The transections, which often affected the alveus of the hippocampal formation, were $0.5-1.0 \mathrm{~mm}$ long in rostrocaudal extent and 3-4 mm long in dorsoventral extent. Because the cannula transections could not follow the exact curvature of the rhinal fissure, TC/LEC connections between a relatively small part in the caudal end of TC and in the rostral end of LEC were probably not accessible for denervation (in total, about one third of the fibers). The mean percentage of fiber lesion for all subgroups of cycloserine- and saline-treated rats was $92 \%$ (range, $85 \%-98 \%$ ), indicating that a total of about $60 \%$ of the fibers between the TC and the LEC were disconnected (Figure 1). The mean percentage of fiber lesion was $90 \%$ (range, $85 \%-94 \%$ ) for the salinetreated subgroups. For the cycloserine-treated subgroups, the mean percentage fiber lesion was $95 \%$ (range, $93 \%$ $98 \%$ ). Among the latter groups, an enlargement of the lesion site was often observed. Such enlargement was much more frequently seen in the left hemisphere $(62 \%)$ than in the right $(4 \%)$. In $12 \%$ of the cases, the lesion enlargement was bilateral. The expansion of the lesion site was unrelated to the time when the rats received injection of cycloserine. Often the lateral ventricle was also enlarged in the animals affected.

\section{Behavior}

The groups from the various treatment categories did not differ significantly in trials to criterion during preoperative acquisition of the discrimination task. During retention, rats from the various categories displayed very different behavior (Figure 3). The subgroups of control rats that received saline on Postoperative Days 0,6 , or 12 did not deviate noticeably from one another and were treated as a single control group. This was also the case for the TC/LEC subgroups that received saline after the 

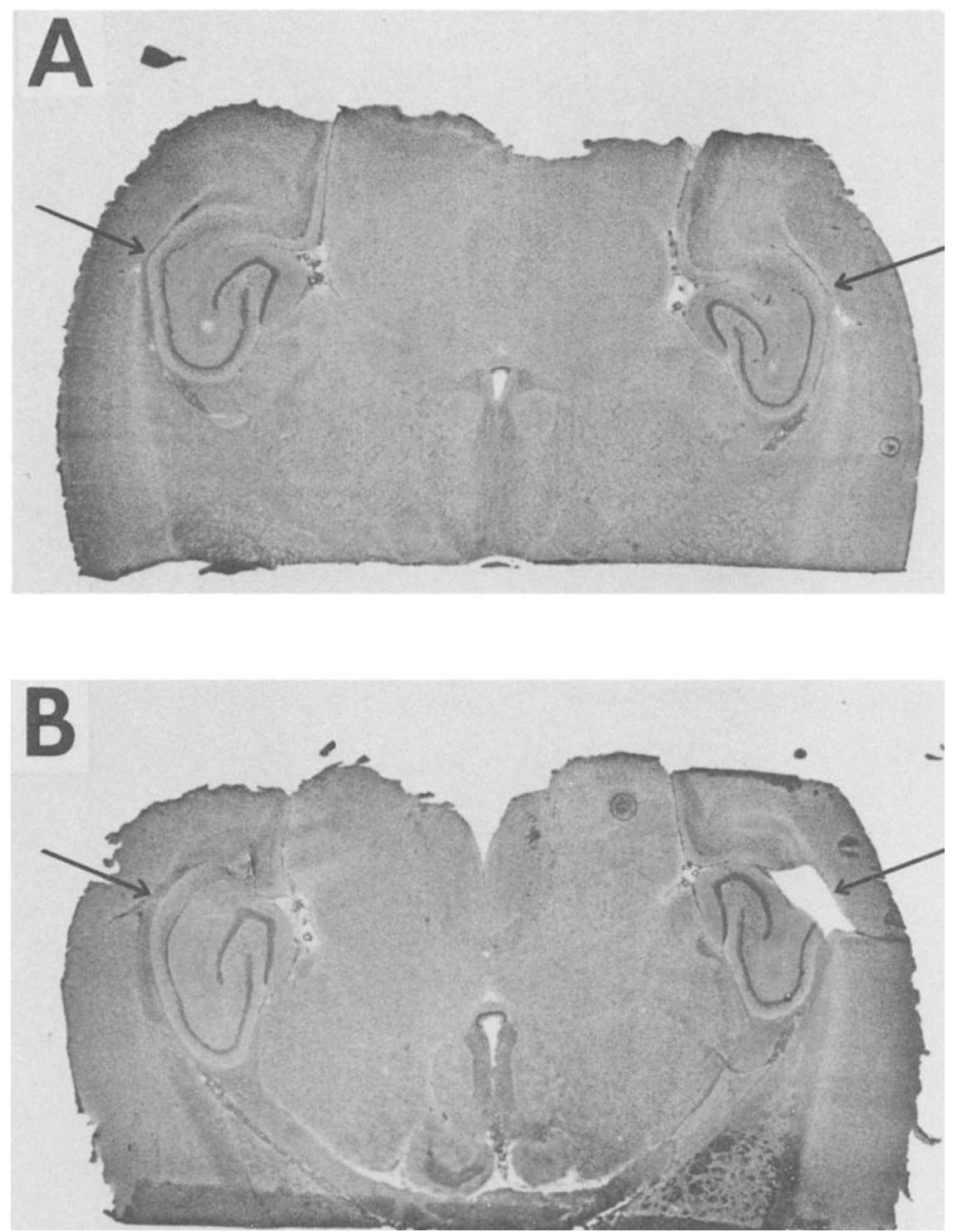

Figure 2. Photographs of horizontal sections showing appearance of lesions (arrows) in rats treated with saline (A) and cycloserine (B).

same postoperative intervals and were similarly treated as a single group. The TC/LEC + saline group committed reliably more errors to reach learning criterion than did the control group $[t(22)=11.40, p<.0001]$. These groups also differed significantly in terms of trials to criterion $[t(22)=9.32, p<.0001]$. The relatively small number of animals in each subgroup $(n=4)$ receiving cycloserine might have reduced the reliability of the ANOVAs. To avoid this problem, the subgroups were pooled into 2-day blocks. Because the performances on Days 0 and 1 were very similar, Day 0 was deleted from the analysis in order to make up 6 pairs of subgroups $(1+2$ to $11+12)$, each consisting of 8 animals. A one-way ANOVA for repeated measures confirmed significant differences among the blocked subgroups in terms of errors $[F(5,7)=7.834, p<.0001]$. Tukey-Kramer multiple-comparison tests showed significant differences among the following pooled subgroups: $1+2$ versus $7+8(p<.001) ; 3+4$ versus $7+8(p<.001) ; 7+8$ versus $9+10(p<.05)$; and $7+8$ versus $11+12(p<$ $.001)$. A one-way ANOVA for repeated measures also confirmed reliable differences among the pooled subgroups in terms of trials $[F(5,7)=8.307, p<.0001]$. Tukey-Kramer multiple comparison tests showed significant differences among the following blocked subgroups: $1+2$ versus $7+8(p<.001) ; 3+4$ versus $7+8$ $(p<.001) ; 5+6$ versus $7+8(p<.05) ; 7+8$ versus $9+10$ $(p<.05)$; and $7+8$ versus $11+12(p<.001)$.

An attempt was made to estimate at what injection intervals cycloserine produced an effect that was significantly weaker than the initial beneficial effects obtained on Days 0 and 1. The error performances of the groups 

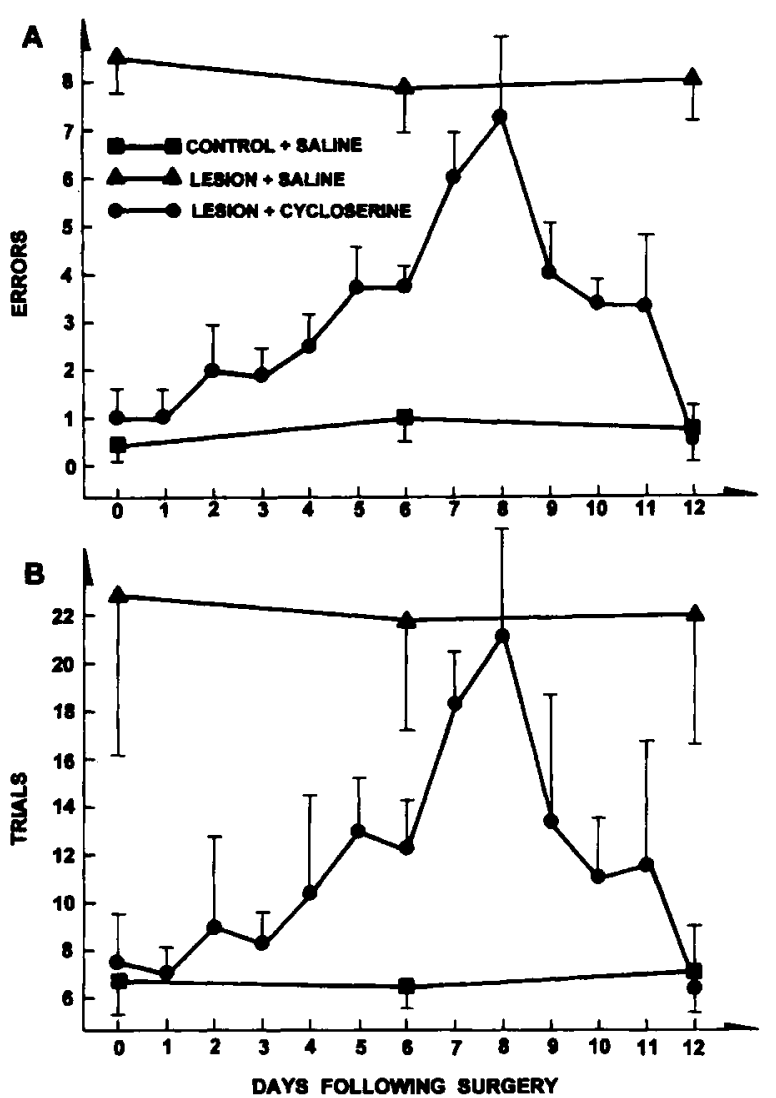

Figure 3. Mean measures $( \pm S E M)$ of retention performance in response to injections in terms of errors (A) and trials (B). The performances of saline-treated control rats and saline-treated lesioned rats are based on three subgroups. The performances of lesioned rats treated with cycloserine are based on 13 subgroups.

that received cycloserine on Days 0 and 1 were pooled to form one reference group. The performance of the group that received injection on Day 4 did not differ reliably from that of the reference group in terms of errors $[t(10)=2.20, p=.110]$, whereas the performance of the group that received injection on Day 5 was reliably different from that of the reference group $[t(10)=4.331$, $p=.001]$. For the number of trials, the performances of the groups that received cycloserine on Days 0 and 1 were pooled to form a single reference group. Group comparisons showed that the performance of rats injected on Day 4 did not differ reliably from that of the reference group $[t(10)=1.49, p=.460]$, whereas the performance of rats injected on Day 5 was significantly different from that of the reference group $[t(10)=4.52, p=$ .012].

\section{DISCUSSION}

The main results from this study show that salinetreated rats with TC/LEC lesions displayed a pronounced retrograde amnesia. However, administration of the glutamatergic partial agonist D-cycloserine re- versed the memory impairment in various degrees, depending on the time of injection between surgery and testing for retention. The variations in effects ranged from complete restoration of the mnemonic function to no effect at all on memory.

The present finding of mitigating effects of cycloserine on retroactive memory may not appear that unexpected in view of previous positive effects on this kind of memory obtained with other agonists such as NMDA, glycine, and AMPA (Myhrer \& Paulsen, 1992). It should be noted, however, that cycloserine is a partial agonist that only has $60 \%$ of the maximal response of glycine (Huettner, 1991). The present results are in agreement with those seen when cycloserine is administered to intact rats. Cycloserine of the same concentration as used in the present study $(3 \mathrm{mg} / \mathrm{kg})$ enhanced memory of a one-trial passive avoidance task when injected i.p. either just before shock or after shock during acquisition and just prior to retrieval (Monahan et al., 1989). In the latter study, the retention time was 1 day, so it was not feasible to test effects of agonists administered at various times between acquisition and retrieval. In subsequent studies, it has been shown that systemic administration of cycloserine can enhance memory performances in lesioned rats, intact mice, and intact rabbits (Flood, Morley, \& Lanthorn, 1992; Quartermain, Mower, Rafferty, Herting, \& Lanthorn, 1994; Schuster \& Schmidt, 1992; Thompson, Moskal, \& Disterhoft, 1992). It has, however, been reported that long-lasting, chronic exposure to the drug may result in desensitization and lack of positive effect (Quartermain et al., 1994).

In the present study, the rats were tested for retention 12 days following surgery. One injection of cycloserine on Postoperative Days 0,1 , or 12 resulted in complete restoration of memory in rats bearing TC/LEC lesions. Injections administered 8 days after surgery were without effect on memory performance. This pattern of effects resembles that seen when glycine ameliorates anterograde amnesia in rats with corresponding lesions (Myhrer et al., 1993). In the latter study, administration of glycine immediately after acquisition or just prior to retention reinstated normal mnemonic function, whereas glycine given midway between acquisition ( 6 days after) and retention had no significant effect on memory. The present results show that the group that received injections on postoperative Day 5 displayed significantly more errors and trials than did the combined group that received injections on Day 0 or 1 . In a previous study with the same test and test procedure as in this study, but with a larger number of rats in each group, the border for significant differences between lesioned rats and controls was located between two and three errors and 8 and 10 trials (Myhrer, 1991).

The action profile of cycloserine is not easy to interpret. It might be speculated whether the profile reflects two distinct mechanisms. Recent findings suggest that different mechanisms are mediating the effects of cycloserine on acquisition and retrieval processes in mice (Quartermain et al., 1994). It appears as if the effects seen for Days 0-8 are associated with declining strength 
of memory engrams with elapse of time. The older the surgically weakened engrams grow, the smaller the possibility of strengthening the capability of these engrams by means of functional mechanisms. The effects seen for Days 9-12 may reflect pharmacological influence of the agonist. The closer cycloserine is administered to the time of retrieval, the better the retention performance. An alternative interpretation might be that cycloserine strengthened engrams when given shortly after training and reactivated them when given shortly before retention testing. The present task is relatively easy to learn, and reacquisition could occur quickly at retention testing.

The finding that cycloserine is able to antagonize memory impairment when administered shortly after surgery suggests that functional mechanisms outlast pharmacological ones. A potential candidate involved in such functional mechanisms may be long-term potentiation (LTP). Cycloserine activates the glycine site at the NMDA receptors. Both NMDA and non-NMDA receptors are most likely involved in LTP (Bekkers \& Stevens, 1989). However, NMDA receptors are required for the induction of LTP, but not for the expression of LTP that involves AMPA receptors. In correspondence with this notion, NMDA receptor antagonists impair acquisition, but not retrieval of previously acquired information (Morris, 1989). Furthermore, AMPA receptor antagonists have been reported to block retrieval of acquired information (Izquierdo, da-Silva, Bueno-e-Silva, Quillfeldt, \& Medina, 1993). The present finding of improved retention when an NMDA agonist is administered after acquisition does not correspond with the pattern of receptor involvement in LTP as outlined above. It cannot, however, be entirely ruled out that LTP might be affected in other ways than those currently known. It is also possible that cycloserine may affect mechanisms of synaptic plasticity other than those involved in LTP. In previous studies of intact mice, administration of cycloserine after acquisition has been shown to improve retention. Such results indicate that in some behavioral tasks, activation of the NMDA complex can facilitate expression of a learned response. However, the mechanisms underlying this facilitation are unclear (Flood et al., 1992; Quartermain et al., 1994).

The finding that cycloserine completely restored memory when injected immediately before retrieval (Day 12) implicates pharmacological activity in the compensatory mechanisms involved. It is also possible that the enhanced performance seen in rats injected on Day 11 is related to the presence of the drug in the system. Cycloserine is a relatively short-acting drug with a half life of only $23 \mathrm{~min}$ in the mouse (Quartermain et al., 1994). However, how long cycloserine remains active at the receptor complex of the rat is not known. If the glycine site of the NMDA receptor is blocked by the antagonist (+)-HA-966 in rats with TC/LEC lesions, the mitigating effect of glycine is prevented. This effect is seen when the animals are injected on Day 0 as well as on Day 12 (Myhrer, 1994). These findings provide evi- dence that both functional and pharmacological mechanisms involve activation of NMDA receptors.

The TC/LEC area seems to be critically involved in preservation of information from the discrimination task. The compensatory effect on memory may have occurred in several different ways. The finding that cycloserine could restore the mnemonic function completely when administered shortly after surgery or immediately prior to retention suggests that the memory engrams interfered with were specific to the present discrimination task. Otherwise, it might have been argued that the agonist caused a general enhancement of compensatory activity in brain areas other than those primarily affected by the lesion. Because TC/LEC lesions only affect about two thirds of the fiber connections between these two areas, cycloserine may possibly act by stimulating glutamatergic activity in residual systems. Alternatively, the agent may trigger compensatory activity in glutamatergic systems outside the TC/LEC area, if these systems process information specific to the present task. It is also possible that agent-induced auxiliary processes in remnant subregions play in concert with compensatory processes in functionally related structures. However, the issue of localizing the mitigating processes is probably most adequately addressed by injecting agonists directly into relevant areas of the brain.

Agonists acting at the glycine receptor site make glutamatergic receptors respond more effectively to endogenous glutamate. For glycine, this is seen as an increased frequency of channel openings and not the current amplitude (Cotman, Monaghan, \& Ganong, 1988). Thus, cycloserine may enhance synaptic efficacy in remnant glutamatergic systems to compensate for the disruptive effects of TC/LEC lesions on memory. Cycloserine acts as a partial agonist during the presence of glycine (Huettner, 1991). However, cycloserine in high doses (>30 mg/ $\mathrm{kg}$ ) has antagonistic properties and exhibits anxiolytic effects in rats (Anthony \& Nevins, 1993). Glycine sites at the NMDA receptors are normally below the saturation point in the hippocampus of rats (Dalkara, Erdemli, Barun, \& Onur, 1992). Thus, NMDA receptor activity may be modulated by agonists acting at the glycine site.

The prevalent enlargement of the lesion site in the left hemisphere seen in cycloserine-treated rats may be related to excitotoxic effects, because the left LEC has been shown to contain higher concentration of glutamate than has the right LEC in our rats (Myhrer et al., 1989). The fact that the lesion enlargement was unrelated to the time of injection is probably associated with the circumstance that the animals lived for several days after testing for retention had been completed. However, the finding of enlarged lesions appeared somewhat unexpected in view of the weaker effect of cycloserine relative to glycine. A similar lesion enlargement, but less prominent, was encountered in rats treated with glycine (Myhrer et al., 1993). A potential explanation of the effects of cycloserine may be that this agent crosses the blood-brain barrier freely (Monahan et al., 1989), whereas 
glycine crosses only slightly (Oldendorf, 1971). Another aspect is that the relative impact of the present dose of 3$\mathrm{mg} / \mathrm{kg}$ cycloserine versus that of $750-\mathrm{mg} / \mathrm{kg}$ glycine in previous studies is not known.

Samples of cortical tissue have been removed surgically from sufferers of Alzheimer's disease for diagnostic purposes. The main findings from such biopsy samples are a loss of corticocortical association fibers and a decreased content of glutamate (Francis et al., 1992). It has been proposed that cycloserine should be tested for clinical efficacy in Alzheimer's disease, because this compound may counteract the cognitive impairment associated with glutamatergic malfunction (Francis, Sims, Procter, \& Bowen, 1993). It has, however, been reported that glycine-dependent binding at the NMDA receptor is considerably reduced in Alzheimer's disease (Steele, Palmer, Stratmann, \& Bowen, 1989). On the other hand, in slices of cortical tissue taken from Alzheimer patients, D-cycloserine facilitates activation of the NMDA receptor-ionophore complex (Chessell, Procter, Francis, \& Bowen, 1991).

The results from the present study suggest that cycloserine is an effective agonist able to reinstate normal memory function in rats with TC/LEC lesions. The TC/LEC specimen has been suggested as a glutamatergic denervation model of Alzheimer's disease (Myhrer, 1993). It is possible, however, that cycloserine may only be helpful early in this disease before glutamatergic systems are severely compromised. Alternatively, cycloserine may be beneficial in age-associated memory impairment accompanying the normal or slightly accelerated aging process. D-Cycloserine appears to be an appropriate pilot compound, and the examination of this compound may permit the development of other potentially useful drugs.

\section{REFERENCES}

AnThony, E. W., \& Nevins, M. E. (1993). Anxiolytic-like effects of $\mathrm{N}$-methyl-D-aspartate-associated glycine receptor ligands in the rat potentiated startle test. European Journal of Pharmacology, 250, 317-324.

BEKKERS, J. M., \& STEVENS, C. F. (1989). NMDA and non-NMDA receptors are co-localized at individual excitatory synapses in cultured rat hippocampus. Nature, 341, 230-233.

Chessell, T. P., Procter, A. W., Francis, P. T., \& Bowen, D. M. (1991). D-Cycloserine, a putative cognitive enhancer, facilitates activation of the $N$-methyl-D-aspartate receptor-ionophore complex in Alzheimer brain. Brain Research, 565, 345-348.

Cotman, C. W., Monaghan, D. T., \& Ganong, A. H. (1988). Excitatory amino acid neurotransmission: NMDA receptors and Hebbtype synaptic plasticity. Annual Review of Neuroscience, 11, 61-80.

Dalkara, T., ERdemli, G., Barun, S., \& ONUR, R. (1992). Glycine is required for NMDA receptor activation: Electrophysiological evidence from intact rat hippocampus. Brain Research, 576, 197-202.

Flood, J. F., Morley, J. E., \& Lanthorn, T. H. (1992). Effect on memory processing by D-cycloserine, an agonist of the NMDA glycine receptor. European Journal of Pharmacology, 221, 249-254.

Francis, P. T., Pangalos, M. N., \& Bowen, D. M. (1992). Animal and drug modelling for Alzheimer synaptic pathology. Progress in Neurobiology, 39, 517-545.
Francis, P. T., Sims, N. R., Procter, A. W., \& Bowen, D. M. (1993). Cortical pyramidal neurone loss may cause glutamatergic hypoactivity and cognitive impairment in Alzheimer's disease: Investigative and therapeutic perspectives. Journal of Neurochemistry, 60, 15891604.

Huettner, J. E. (1991). Competitive antagonism of glycine at the $N$-methyl-D-aspartate (NMDA) receptor. Biochemical Pharmacology, 41, 9-16.

IZquierdo, I., DA-Silva, R. C., Bueno-e-Silva, M., Quillfeldt, J. A., \& MEDina, J. H. (1993). Memory expression of habituation and of inhibitory avoidance is blocked by CNQX infused into the entorhinal cortex. Behavioral \& Neural Biology, 60, 5-8.

Monahan, J. B., Handelmann, G. E., Hood, W. F., \& Cordi, A. A. (1989). D-Cycloserine, a positive modulator of the $\mathrm{N}$-methyl-Daspartate receptor, enhances performance of learning tasks in rats. Pharmacology, Biochemistry \& Behavior, 34, 649-653.

MORRIS, R. G. M. (1989). Synaptic plasticity and learning: Selective impairment of learning in rats and blockade of long-term potentiation in vivo by the $N$-methyl-D-aspartate receptor antagonist AP5. Journal of Neuroscience, 9, 3040-3057.

MYHRER, T. (1991). Retroactive memory of a visual discrimination task in the rat: Role of temporal-entorhinal cortices and their connections. Experimental Brain Research, 84, 517-524.

MYHRER, T. (1992). Selective lesions in the temporal-hippocampal region of the rat: Effects on acquisition and retention of a visual discrimination task. Behavioral \& Neural Biology, 58, 8-15.

MYHRER, T. (1993). Animal models of Alzheimer's disease: Glutamatergic denervation as an alternative approach to cholinergic denervation. Neuroscience \& Biobehavioral Reviews, 17, 195-202.

MYHRER, T. (1994). Evidence for activation of NMDA receptors when memory function is reinstated in rats with glutamatergic temporal systems disrupted. Brain Research, 662, 263-267.

MYHRER, T., IVERSEN, E. G., \& FonNUM, F. (1989). Impaired reference memory and reduced glutamergic activity in rats with temporoentorhinal connections disrupted. Experimental Brain Research, 77, 499-506.

Myhrer, T., Johannesen, T. S., \& SpikKerud, E. (1993). Restoration of mnemonic function in rats with glutamergic temporal systems disrupted: Dose and time of glycine injections. Pharmacology, Biochemistry \& Behavior, 45, 519-525.

MYHRER, T., \& NAVDAL, G. A. (1989). The temporal-hippocampal region and retention: The role of temporo-entorhinal connections in rats. Scandinavian Journal of Psychology, 30, 72-80.

MYHRER, T., \& PAULSEN, R. E. (1992). Memory dysfunction following disruption of glutamergic systems in the temporal region of the rat: effects of agonistic amino acids. Brain Research, 599, 345-352.

OLDENDORF, W. H. (1971). Brain uptake of radio labeled amino acids, amines, and hexoses after arterial injection. American Journal of Physiology, 221, 1629-1639.

Quartermain, D., Mower, J., Rafferty, M. F., Herting, R. L., \& LANTHORN, T. M. (1994). Acute but not chronic activation of the NMDA-coupled glycine receptor with D-cycloserine facilitates learning and retention. Eumopean Journal of Pharmacology, 257, 7-12.

SCHUSTER, G. M., \& SCHMIDT, W. J. (1992). D-Cycloserine reverses the working memory impairment of hippocampal-lesioned rats in a spatial learning task. European Journal of Pharmacology, 224, 97-98.

Steele, J. E., Palmer, A. M., Stratmann, G. C., \& Bowen, D. M. (1989). The $N$-methyl-D-aspartate receptor complex in Alzheimer's disease: Reduced regulation by glycine but not zinc. Brain Research, 500, 369-373.

Thompson, L. T., Moskal, J. R., \& Disterhoft, J. F. (1992). Hippocampus-dependent learning facilitated by a monoclonal antibody or D-cycloserine. Nature, 359, 638-641.

(Manuscript received January 26, 1995; revision accepted for publication May 10, 1995.) 